\title{
Estudios cariotípicos en especies de Gilliesieae Lindl. (Gilliesioideae- Alliaceae) de Chile central
}

\section{Karyotypic studies in species of Gilliesieae Lindl. (Gilliesioideae-Alliaceae) from central Chile}

\author{
Inelia Escobar*, Eduardo Ruiz \& Carlos Baeza
}

Departamento de Botánica, Facultad de Ciencias Naturales y Oceanográficas, Universidad de Concepción, Casilla 160-C, Concepción, Chile.

*iescobar@udec.cl

\begin{abstract}
RESUMEN
La tribu Gilliesieae Lindl. es parte del grupo sudamericano de Alliaceae, caracterizada por presentar una alta diversificación floral, desplegando diferentes grados de simetría bilateral, variación del número de tépalos y estambres, y presencia de apéndices florales, con altos niveles de endemismo principalmente en la zona del clima mediterráneo de Chile central. Desde el punto de vista citológico, sólo se conoce la dotación cromosómica de tres especies. Este estudio presenta el cariotipo de 10 especies representantes de los géneros Ancrumia, Gethyum, Gilliesia, Miersia, Solaria y Speea. Se indican los primeros reportes para 7 taxones: Ancrumia cuspidata, Gilliesia graminea, G. montana, Miersia minor, M. leporina, M. tenuiseta y Speea humilis. Se observan tres set cromosómicos, Miersia spp. y Speea $2 \mathrm{n}=12(10 \mathrm{~m}+2 \mathrm{t})$, M. chilensis $2 \mathrm{n}=20$ $(2 \mathrm{~m}+18 \mathrm{t})$ y Ancrumia, Gethyum, Gilliesia y Solaria $2 \mathrm{n}=14(4 \mathrm{~m}+4 \mathrm{sm}+6 \mathrm{t})$. Las especies estudiadas muestran cariotipos diploides, con largos cromosomas de hasta $22 \mu \mathrm{m}$, frecuentemente del tipo metacéntrico y telocéntrico y un conservado número fundamental $(\mathrm{NF}=11)$. Los resultados sugieren que translocaciones Robertsonianas podrían jugar un rol importante en la evolución del cariotipo en la tribu y subfamilia.
\end{abstract}

Palabras clave: Cariotipos, número cromosómico, translocaciones Robertsonianas, físiones céntricas, endemismo.

\begin{abstract}
The tribe Gilliesieae Lindl. belong to the South American group of Alliaceae, is characterized by high floral diversification, displaying different degree of bilateral symmetry, varied number of tepals and stamens and floral appendages, with high levels of endemism mainly in the Mediterranean climate zone of central Chile. Cytologically chromosome numbers only of three species are known. In the present study, the karyotype of ten species corresponding to the genera Ancrumia, Gethyum, Gilliesia, Miersia, Solaria and Speea, are documented. First reports are provided for seven taxa: Ancrumia cuspidata, Gilliesia graminea, G. montana, Miersia minor, M. leporina, M. tenuiseta y Speea humilis. Three chromosomal sets are observed, Miersia spp. and Speea with $2 \mathrm{n}=12(10 \mathrm{~m}+2 \mathrm{t})$, M. chilensis with $2 \mathrm{n}=20(2 \mathrm{~m}+18 \mathrm{t})$ and Ancrumia, Gethyum, Gilliesia and Solaria with $2 \mathrm{n}=14(4 \mathrm{~m}+4 \mathrm{sm}+6 \mathrm{t})$. The studied species are diploid and showed large chromosomes, some of them larger than $22 \mu \mathrm{m}$, frequently metacentric or telocentric, and with a conserved fundamental number $(\mathrm{FN}=11)$. The results suggest that Robertsonian exchanges could play a major role in the evolution of the tribe and subfamily.
\end{abstract}

KeYwords: Karyotypes, chromosome number, Robertsonian exchanges, centric fission, endemism.

\section{INTRODUCCION}

Los representantes sudamericanos de Alliaceae Borkh se encuentran reunidos en la subfamilia Gilliesioideae (Lindl.) Arn., la cual a su vez está formada por dos tribus, una ampliamente diversificada y distribuida en la región, reconocida informalmente como tribu Ipheieae, que comprende los géneros Nothoscordum Kunth, Ipheion Raf.,
Tristagma Poepp., Leucocoryne Lindl., Zoellnerallium Crosa y la tribu Gilliesieae Lindl. mayormente diversa en Chile central, y que comprende los géneros Gethyum Phil., Gilliesia Lindl., Miersia Lindl., Speea Loes., Solaria Phil. Trichlora Baker y Schickendanziella Speg., que reúnen entre 9 y 24 especies dependiendo del autor (Fay \& Chase 1996, Rahn 1998, Muñoz 2000, Ravenna 2000 a-c, 2005 a-b, Souza et al. 2010). Gilliesieae es una tribu de geófitas 
bulbosas, diferenciada del resto de los representantes de la familia por sus variados grados de simetría floral bilateral, variación en el número de tépalos y estambres, y novedosos apéndices florales (Rudall et al. 2002). Esta se distribuye principalmente en la zona del clima mediterráneo de Chile $\left(30^{\circ}-36^{\circ} \mathrm{S}\right)$, área considerada dentro del hotspot de biodiversidad chileno, pero también con algunos representantes en Argentina, Perú y Bolivia (Myers et al 2000, Arroyo et al. 2004, Fay \& Hall 2007, Escobar et al. 2010, Negritto et al. 2010).

A diferencia del amplio conocimiento citológico que se tiene del resto de los representantes de la subfamilia (Crosa 1972, 1975, 1981, 1988, Araneda et al. 2004, Salas \& Manzur 2004, Meric \& Dane 2005, Souza et al. 2009, Souza et al. 2010), estudios citológicos en los representantes de Gilliesieae son escasos y sólo han provisto información acerca de la dotación cromosómica de tres especies. Cave \& Bradley (1943) señalaron el número diploide, $2 \mathrm{n}=20 \mathrm{y}$ 21 para Miersia chilensis Lindl., posteriormente Goldblatt (1976) confirmó el $2 \mathrm{n}=20$ para esta misma especie, con un par metacéntrico y nueve pares de cromosomas telocéntricos, sin embargo, Del Poso \& Zöllner (1976) indican un número cromosómico $2 \mathrm{n}=12$ para esta misma especie. En Gethyum atropurpureum Phil., el número cromosómico es $2 \mathrm{n}=14$, con 2 pares metacéntricos y 3 pares telocéntricos (Goldblatt 1976), y en Solaria attenuata Ravenna (sinonimizada bajo Solaria miersioides Phil. por Muñoz, 2000) 2n=14, con 2 pares submetacéntricos, 3 pares subtelocéntricos y 2 pares acrocéntricos (Ravenna 1967, Rahn 1998).

El número cromosómico es una útil herramienta en sistemática, dado la simplicidad para obtener información substancial acerca del genoma de una especie, sin embargo sólo tienen implicancias en la filogenia y evolución del cariotipo la variación de éste por procesos de disploidía y poliploidía (Guerra 2000, 2008). En este contexto los reordenamientos estructurales (ej. translocaciones Robertsonianas) y la duplicación o multiplicación de un complemento cromosómico completo constituyen importantes procesos. Por lo cual, la incorporación de evidencia citológica resulta fundamental para esclarecer las relaciones filogenéticas dentro de la tribu y subfamilia.

Frecuentemente, un conservado número fundamental (NF) junto a la presencia de cromosomas metacéntricos y telocéntricos, sugieren procesos de reorganización Robertsoniana entre los diferentes cariotipos, donde cambios en el número cromosómico son generados por fusión o fisión de cromosomas (Duncan \& MacLeod 1950, Cox et al. 1998, Tamura 1995, Pires et al. 2006). Tamura (1995) señaló que rearreglos Robertsonianos ocurren en Alliaceae s.l., siendo reconocido Nothoscordum como un clásico ejemplo de evolución cariotípica, con un cariotipo bimodal originado por fusiones y fisiones céntricas, exhibiendo cromosomas metacéntricos con una longitud equivalente a la suma de dos telocéntricos (Jones 1998, Pires et al. 2006, Souza et al.
2009). A su vez en Gilliesieae, Goldblatt (1976) señala que los cariotipos de Miersia chilensis y Gethyum atropurpureum responden claramente a este tipo de procesos, postulando que la dirección del cambio cromosómico en estas dos especies relacionadas haya sido a la fusión de cromosomas y por ende disminución en el número de éstos. De este modo, G. atropurpureum con cromosomas metacéntricos y telocéntricos y morfológicamente con sólo tres estambres sería más especializado que los representantes de $M$. chilensis, la cual presenta mayoritariamente cromosomas telocéntricos y seis estambres Goldblatt (1976).

En el presente trabajo se estudió el cariotipo para 10 especies de la tribu Gilliesieae que crecen en Chile central, con el objetivo de determinar la dotación cromosómica y proveer información cariológica que ayude a inferir la evolución cromosómica del grupo y esclarecer las relaciones de parentesco en el contexto de recientes investigaciones filogenéticas (Escobar 2012).

\section{MATERIALES Y MÉTODOS}

\section{Material VEGetal}

Se estudiaron 70 individuos de 17 poblaciones correspondientes a 10 de las 17 especies actualmente reconocidas para la tribu (Escobar 2012), representando a todos los géneros que crecen en Chile (Tabla I). Los bulbos fueron cultivados en el invernadero del Departamento de Botánica de la Universidad de Concepción y el material de referencia depositado en el Herbario de la Universidad de Concepción (CONC), acrónimos sensu Holmgren et al. (1990).

\section{OBTENCIÓN DE CARIOTIPOS}

Se extrajo puntas de raíces $(8-10 \mathrm{~mm}$ de longitud), las que fueron pre-tratadas en una solución $2 \mathrm{mM}$ de 8 hidroxiquinoleina durante $24 \mathrm{~h}$ a $4^{\circ} \mathrm{C}$, para una posterior fijación del material en una solución de etanol-ácido acético (3:1) a $4^{\circ} \mathrm{C}$. Posteriormente, se realizó una hidrólisis ácida con $\mathrm{HCl} 0,5 \mathrm{~N}$ durante 25 minutos a $45^{\circ} \mathrm{C}$, lavando posteriormente reiteradas veces con agua destilada, finalmente, se tiñó el ápice de la raíz con una gota de orceína acética al $1 \%$ para el posterior aplastado (Baeza et al. 2000, con modificaciones). El conteo e interpretación de los cromosomas se realizó usando un microscopio Axioskop Zeiss con cámara fotográfica incluida. Se consideró un mínimo de 10 placas metafásicas por especie, incluyendo entre 4 y 12 individuos, dependiendo de la disponibilidad de material. Los cromosomas fueron medidos con el programa MicroMeasure 3.3 (Reeves 2001) y clasificados de acuerdo a la nomenclatura propuesta por Levan et al., (1964), designados por la posición del centrómero: 1.0-1.7 (metacéntrico: m), 1.7-3.0 (submetacéntrico; sm), 3.0-7.0

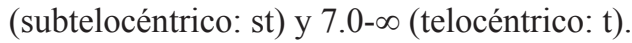


TABLA I. Especies de Gilliesieae Lindl. citológicamente estudiadas y localidades de recolección del material vegetal. Al final de cada localidad se señala la identificación de las poblaciones.

TABLE I. Species cytologically studied of Gilliesieae Lindl. and locations of collection of plant material. At the end of each location indicates the population identification.

\begin{tabular}{|c|c|}
\hline ESPECIES ESTUDIADAS & ORIGEN DEL MATERIAL ESTUDIADO \\
\hline Ancrumia cuspidata Baker & $\begin{array}{l}\text {-Región de Coquimbo. Prov. Limarí. Parque Nacional Fray Jorge, cerca de la administración. } \\
256 \text { m. } 30^{\circ} 39^{\prime} \text { S } 71^{\circ} 40^{\prime} \text { W. 7-IX-2006. I. Escobar, A. Marticorena, M. Negritto \& E. Ruiz } \\
12 \text { (CONC). Pobl.12. }\end{array}$ \\
\hline Gethyum atropurpureum Phil. & $\begin{array}{l}\text {-Región Metropolitana. Prov. Santiago. Peñalolén. Quebrada Nido de Águila. } 1132 \text { m. } \\
33^{\circ} 28^{\prime} 33,6 \text { "S 70³0’03,5”W. 19-VIII-2007. I. Escobar } 44 \text { \& N. García (CONC). Pobl. } \\
44 .\end{array}$ \\
\hline Gilliesia graminea Lindl. & $\begin{array}{l}\text {-Región de Valparaíso. Prov. Quillota. Parque Nacional La Campana, sector Palmas de } \\
\text { Ocoa. } 435 \text { m. } 32^{\circ} 56^{\prime} \text { N } 71^{\circ} 04^{\prime} \text { W. 10-IX-2006. I. Escobar, A. Marticorena, M. Negritto \& E. } \\
\text { Ruiz } 18 \text { (CONC). Pobl. 18. } \\
\text {-Región Metropolitana. Prov. Melipilla. Alhué, camino al cerro Talamí, cercano a la } \\
\text { estación de electricidad. } 600 \text { m. } 34^{\circ} 06^{\prime} \text { 'S } 71^{\circ} 00^{\prime} \text { W. 06-IX-2007. I. Escobar } 94 \text { (CONC). } \\
\text { Pobl. } 94 .\end{array}$ \\
\hline Gilliesia montana Poepp. & $\begin{array}{l}\text {-Región del Maule. Prov. Cauquenes. Reserva Nacional Los Ruiles de Empedrado. } 350 \text { m. } \\
\text { 35³7’41”S 72²0’36”W. 31-VIII-2007. I. Escobar } 56 \text { (CONC). Pobl. } 56 .\end{array}$ \\
\hline Miersia chilensis Lindl. & 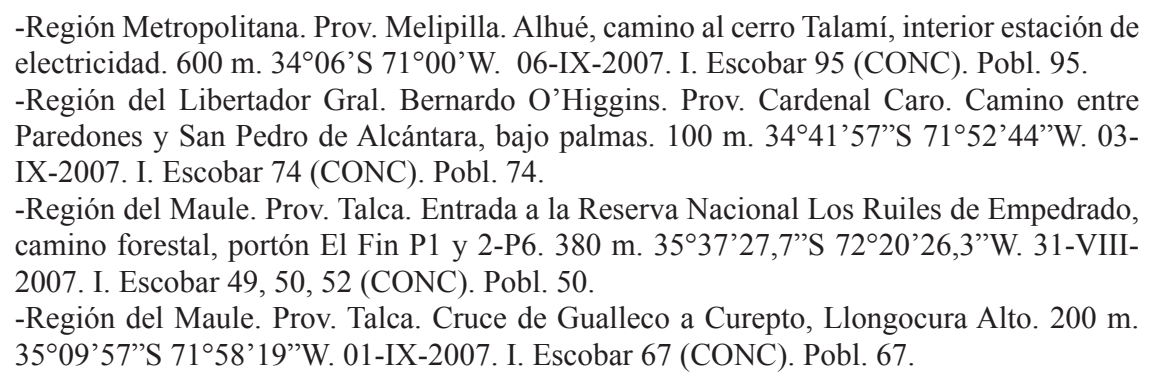 \\
\hline Miersia leporina Ravenna & $\begin{array}{l}\text {-Región de Valparaíso. Prov. Quillota. La Campana, por Ocoa, camino hacia la cascada, } \\
\text { cerca del mirador. } 535 \mathrm{~m} .32^{\circ} 56 \text { 'S } 71^{\circ} 04^{\prime} \text { W. 9-IX-2006. I. Escobar, A. Marticorena, M. } \\
\text { Negritto \& E. Ruiz } 23 \text { (CONC). Pobl. 23. }\end{array}$ \\
\hline Miersia minor Kunth & $\begin{array}{l}\text {-Región del Libertador Gral. Bernardo O’Higgins. Prov. Cardenal Caro. Tanumé Alto, } \\
\text { Centro Experimental Tanumé, sector Los Quillayes, Cerro Cruz de Los Muertos, sendero a } \\
\text { Quebrada Honda. } 220 \text { m. } 34^{\circ} 11^{\prime} 02,8^{\prime} \text { 'S } 71^{\circ} 533^{\prime} 53^{\prime} \text { W. 03-IX-2007. I. Escobar } 75 \text { (CONC). } \\
\text { Pobl. } 75 . \\
\text {-Región del Biobío. Prov. Nuble. Cerro Cayumanque, camino a las antenas de la cumbre, } \\
\text { ladera camino lateral. } 600 \text { m. } 36^{\circ} 41^{\prime} S 72^{\circ} 30^{\prime} \text { W. 31-VII-2007. I. Escobar } 40 \text { (CONC). } \\
\text { Pobl. } 40 .\end{array}$ \\
\hline Miersia tenuiseta Ravenna & 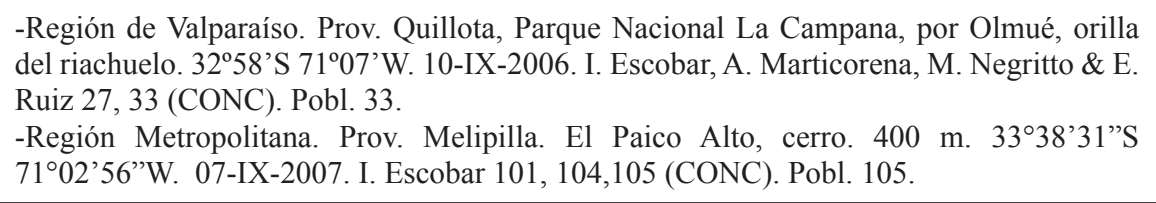 \\
\hline Solaria. miersioides Phil. & $\begin{array}{l}\text {-Región del Maule. Prov. Talca, sector Los Álamos-Cipreses, bajo bosque de Nothofagus } \\
\text { obliqua y N. dombeyi. } 1000 \text { m. 3548'40”S 7048'57,5”W. 18-X-2007. I. Escobar } 137 \\
\text { (CONC). Pobl. } 137 .\end{array}$ \\
\hline Speea humilis (Phil.) Loes. ex K. Krause & 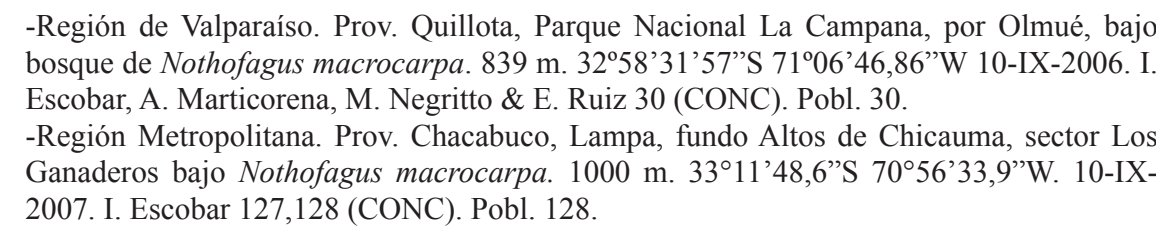 \\
\hline
\end{tabular}




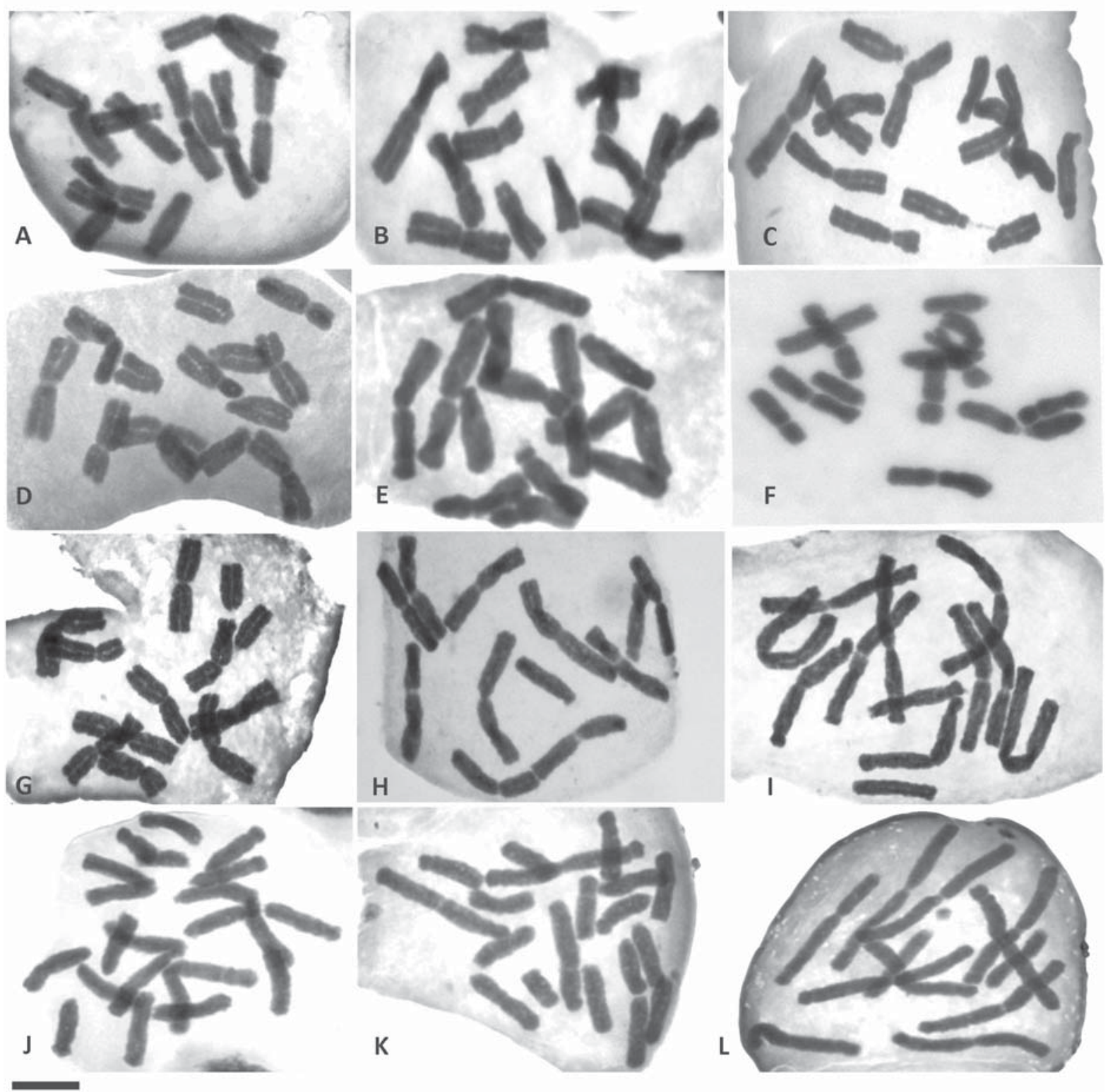

Figura 1. Chromosomas mitóticos de las especies de Gilliesieae estudiadas. A. Ancrumia cuspidata, B. Gethyum atropurpureum, C-D. Gilliesia graminea, E. G. montana, F. Solaria miersioides (fotografía de LGR Souza), G. Miersia tenuiseta, H. M. leporina, I. Speea humilis, J-K. M. chilensis, L. M. minor. Escala=10 $\mu \mathrm{m}$.

FIgURE 1. Mitotic metaphases of studied Gilliesieae species. A. Ancrumia cuspidata, B. Gethyum atropurpureum, C-D. Gilliesia graminea, E. G. montana, F. Solaria miersioides (by LGR Souza), G. Miersia tenuiseta, H. M. leporina, I. Speea humilis, J-K. M. chilensis, L. M. minor. Bars $=10 \mu \mathrm{m}$.

\section{ANÁLISIS DE LOS CARIOTIPOS}

Para cada población se analizó el número, morfología y tamaño de los cromosomas (LT). Se determinó el índice de asimetría del cariotipo (As K\%) descrito por Arano (1963) y Arano \& Saito (1980), expresado como la razón entre la sumatoria de la longitud de los brazos largos de cromosomas individuales y la longitud haploide total del complemento cromosómico. Se estimó el valor R (razón de la longitud relativa del par de cromosoma más largo dividido por el par más corto en el set cromosomal). Para una mejor comparación de los cariotipos se calculó la longitud total del complemento haploide (LTC, $\mu \mathrm{m})$. Representaciones gráficas y procesamiento de imágenes fueron realizadas con el programa Paint Shop Pro 7. 


\section{RESULTADOS}

La fórmula cariotípica, número cromosómico y tamaño de los cromosomas señalados en el presente estudio corresponden a los primeros reportes para siete representantes de la tribu: Ancrumia cuspidata Baker, Gilliesia graminea Lindl., G. montana Poepp., Miersia minor Kunth, M. leporina Ravenna, M. tenuiseta Ravenna y Speea humilis (Phil.) Loes. ex K. Krause (Tabla II, Fig. 2). En las placas analizadas, correspondientes a las especies con más de una población, Gilliesia graminea (2), Miersia chilensis (4), M. minor (2), M. tenuiseta (2), Speea humilis (2) no se observaron diferencias cariotípicas intraespecíficas.

El análisis del cariotipo en la tribu reveló tres complementos cromosómicos diferentes $2 \mathrm{n}=12(10 \mathrm{~m}+2 \mathrm{t})$, $2 n=14(4 m+4 s m+6 t)$ y $2 n=20 \quad(2 m+18 t)$, todos con el mismo número fundamental, $\mathrm{NF}=11 \mathrm{y}$ grandes cromosomas (5-22 $\mu \mathrm{m})$ variando su morfología entre metacéntricos, submetacéntricos y telocéntricos (Tabla II, Fig. 1-3). Las especies estudiadas de Ancrumia, Gethyum, Gilliesia y Solaria presentan un número diploide $2 \mathrm{n}=14$, con un cariotipo formado por dos pares de cromosomas metacéntricos, dos pares submetacéntricos y tres pares telocéntricos. El índice de asimetría (As K\%) varió de 68 a 72,5\% y la longitud cromosómica desde 5 a $14 \mu \mathrm{m}$ (Fig. 3). Por otro lado, las especies de Miersia (con excepción de M. chilensis) y Speea, presentan un $2 \mathrm{n}=12$, con un cariotipo formado por largos cromosomas, con cinco pares metacéntricos de entre $13 \mathrm{y}$ $22 \mu \mathrm{m}$ de longitud y dos pares telocéntricos, de 6,5 a $12 \mu \mathrm{m}$ y valores de As $\mathrm{K} \%$, entre 57,8 y 58,5. Adicionalmente, fue posible observar en algunas placas metafásicas de $M$. minor un pequeño satélite. El número cromosómico para Miersia chilensis es de $2 \mathrm{n}=20$, con un cariotipo bimodal altamente asimétrico (As $\mathrm{K} \%$ 83,2), con un par de cromosomas metacéntricos de $20 \mu \mathrm{m}$ y nueve pares telocéntricos, de entre 6 y $12 \mu \mathrm{m}$ (Fig. 3).

EnAncrumiacuspidata, Gethyumatropurpureum, Gilliesia graminea, G. montana y Solaria miersioides, se observa una alta similitud del tamaño relativo de sus brazos cortos y largos para los tres tipos de cromosomas encontrados, sin embargo en Ancrumia cuspidata el par III muestra variación entre metacéntrico y submetacéntrico (Fig. 1 A, 2, 3), de acuerdo a la clasificación propuesta por Levan et al. (1964). Además, la longitud de cada par cromosómico difiere muy poco entre las especies mencionadas (Fig. 2, 3), variando la longitud del complemento cromosómico haploide de 56,5 a $68,5 \mu \mathrm{m}$, siendo Ancrumia cuspidata el taxón con los cromosomas de mayor longitud (Tabla II). En los representantes de Miersia spp. y Speea, la longitud del complemento cromosómico haploide varía de 86,5 a $104 \mu \mathrm{m}$.

TABLA II. Características del cariotipo de las diez especies de Gilliesieae Lindl. estudiadas. Número diploide (2n), fórmula cariotípica de acuerdo a Levan et al. (1964). As K\%= índice de asimetría del cariotipo (Arano 1963, Arano \& Saito 1980), valor R= razón del par de cromosomas más largo/ par más corto, LTC $(\mu \mathrm{m})=$ longitud total de los cromosomas haploides. * = Primer reporte.

TABLE II. Karyotype characteristics from ten studied species of Gilliesieae Lindl. Diploid number (2n), karyotype formula according to Levan et al. (1964), As K\%= karyotype asymmetry index (Arano 1963, Arano \& Saito 1980), R= ratio longest pair of chromosome/shortest pair, LTC $(\mu \mathrm{m})=$ total haploid chromosome length. $*=$ first record.

\begin{tabular}{|c|c|c|c|c|c|}
\hline EsPecies & $2 n$ & FóRMULA CARIOTÍPICA & As $\mathrm{K} \%$ & $\begin{array}{c}\text { VALOR } \\
\text { R }\end{array}$ & $\mathrm{LTC}(\mu \mathrm{m})$ \\
\hline Ancrumia cuspidata* & 14 & $4 m+4 s m+6 t$ & 68,0 & 2,1 & 68,5 \\
\hline Gethyum atropurpureum & 14 & $4 m+4 s m+6 t$ & 69,6 & 2,0 & 62,0 \\
\hline Solaria miersioides & 14 & $4 m+4 s m+6 t$ & 68,0 & 2,2 & 56,5 \\
\hline Gilliesia graminea* & 14 & $4 m+4 s m+6 t$ & 72,5 & 2,0 & 65,5 \\
\hline Gilliesia montana * & 14 & $4 m+4 s m+6 t$ & 69,4 & 2,1 & 57,5 \\
\hline Miersia chilensis & 20 & $2 \mathrm{~m}+18 \mathrm{t}$ & 83,2 & 3,1 & 104 \\
\hline Miersia tenuiseta $*$ & 12 & $10 \mathrm{~m}+2 \mathrm{t}$ & 58,2 & 2,3 & 102 \\
\hline Miersia minor $*$ & 12 & $10 m+2 t$ & 57,8 & 2,2 & 86,5 \\
\hline Miersia leporina * & 12 & $10 m+2 t$ & 58,5 & 1,9 & 99,0 \\
\hline Speea humilis * & 12 & $10 \mathrm{~m}+2 \mathrm{t}$ & 57,9 & 2,0 & 86,5 \\
\hline
\end{tabular}


Estudios cariotípicos en Gilliesieae Lindl.: EscoBAR, I. ET AL.
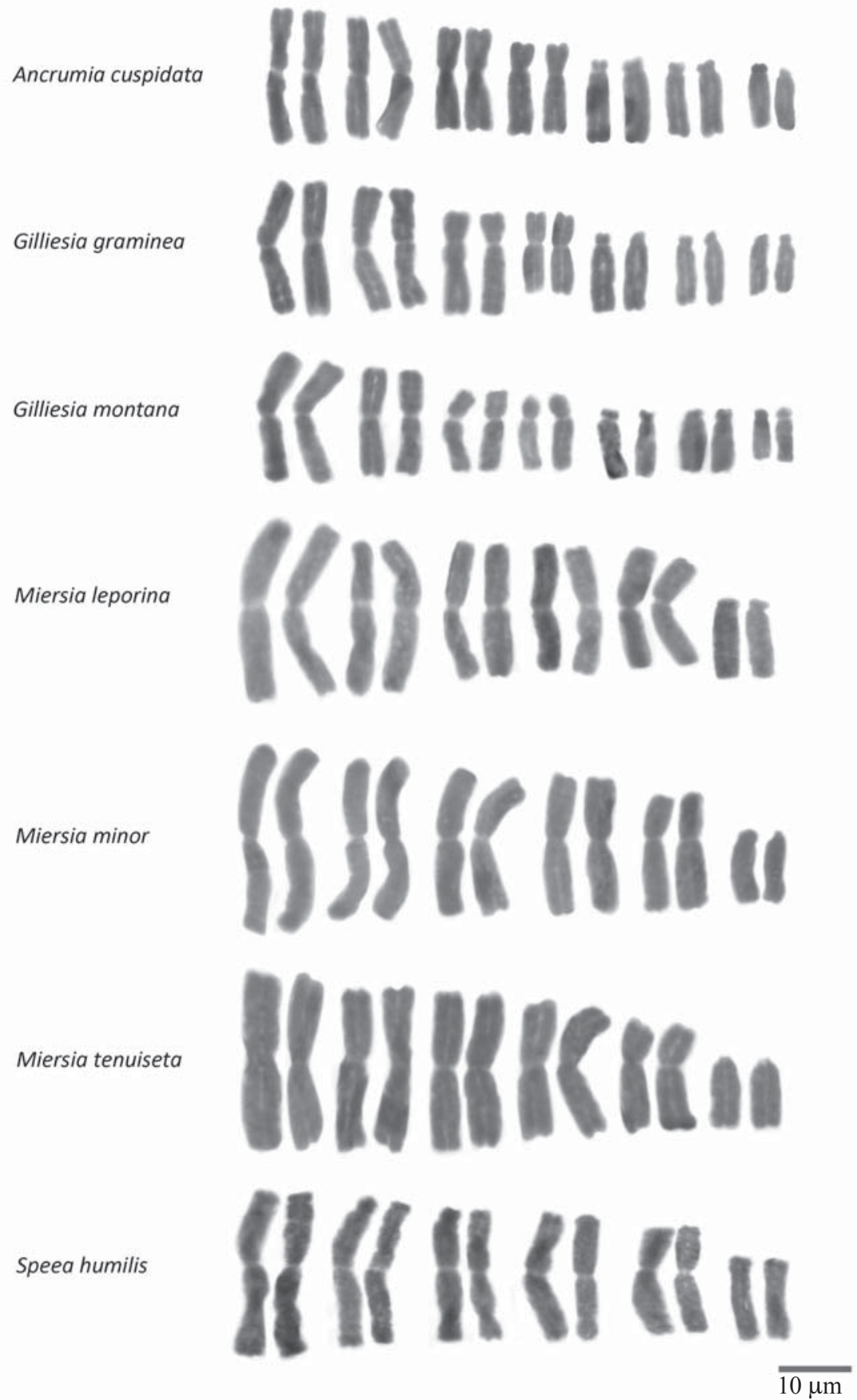

Figura 2. Cariotipos de Ancrumia cuspidata, Gilliesia graminea, G. montana, Miersia leporina, M. minor, M. tenuiseta y Speea humilis. Los cromosomas se han ordenado de acuerdo a su tamaño decreciente. Escala=10 $\mu \mathrm{m}$.

Figure 2. Karyotypes of Ancrumia cuspidata, Gilliesia graminea, G. montana, Miersia leporina, M. minor, M. tenuiseta y Speea humilis. Chromosomes were ordered by decreasing size. Bars $=10 \mu \mathrm{m}$. 
Gethyum atropurpureum

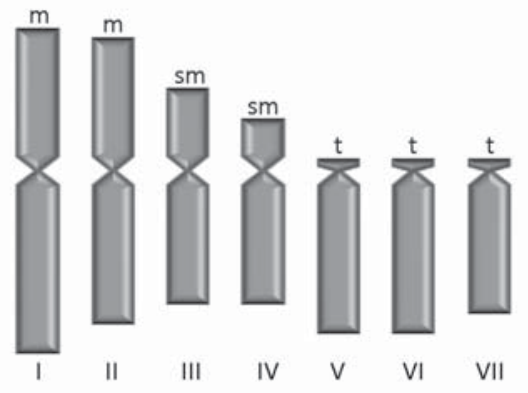

\begin{tabular}{|l|l|l|l|l|l|l|l|}
\hline BU(4) & $6,02 \pm 0,69$ & $4,98 \pm 0,14$ & $4,35 \pm 0,59$ & $4,45 \pm 0,09$ & $5,42 \pm 0,12$ & $5,12 \pm 0,24$ & $4,66 \pm 0,12$ \\
\hline
\end{tabular} \begin{tabular}{|l|l|l|l|l|l|l|l|}
\hline $\mathrm{BC}(90)$ & $4,79 \pm 0,83$ & $4,27 \pm 0,11$ & $2,64 \pm 0,01$ & $1,87 \pm 0,11$ & $0,5650,10$ & $0,45 \pm 0,04$ & $0,41 \pm 0,10$ \\
\hline
\end{tabular}

\begin{tabular}{|l|c|c|c|c|c|c|c|}
\hline $\operatorname{LR}(\mathrm{m})$ & 10,81 & 9,25 & 6,99 & 6,33 & 5,98 & 5,57 & 5,07 \\
\hline $\operatorname{IT}(\mu \mathrm{m})$ & 12,0 & 11,0 & 9,5 & 8,5 & 8,0 & 7,0 & 6,0 \\
\hline$R$ & 1,2 & 1,1 & 1,8 & 2,2 & 8,6 & 9,8 & 10,5 \\
\hline
\end{tabular}

Gilliesia graminea

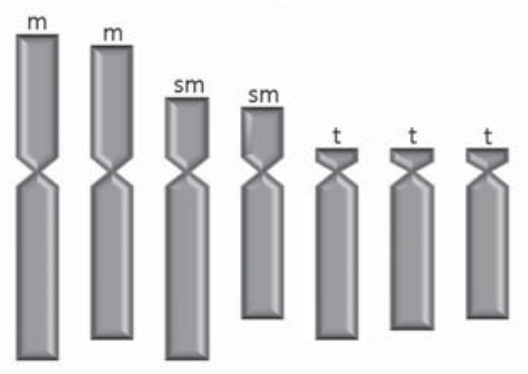

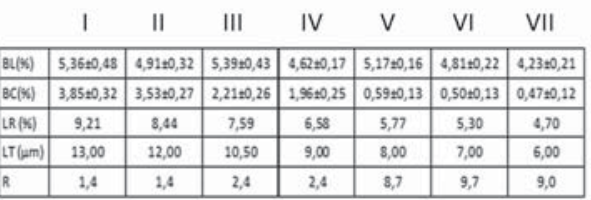

Solaria miersioides

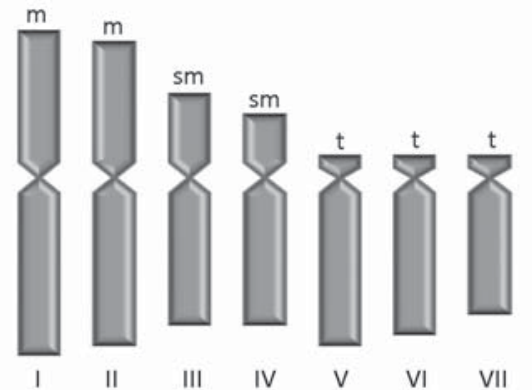

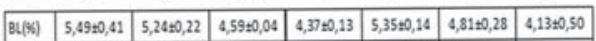
\begin{tabular}{|l|l|l|l|l|l|l|l|l|}
\hline $\operatorname{BC}(\$)$ & $4,75 \pm 0,06$ & $4,61 \pm 0,54$ & $2,75 \pm 0,54$ & $2,09 \pm 0,18$ & $0,63 \pm 0,08$ & $0,62 \pm 0,05$ & $0,59 \pm 0,06$ \\
\hline
\end{tabular}

\begin{tabular}{|l|c|c|c|c|c|c|c|}
\hline $\operatorname{LR}(\mathrm{s})$ & 10,24 & 9,84 & 7,34 & 6,45 & 5,98 & 5,42 & 4,71 \\
\hline $\operatorname{LT}(\mathrm{um})$ & 12,00 & 11,50 & 8,50 & 7,00 & 6,50 & 6,00 & 5,00 \\
\hline$R$ & 1,2 & 1,1 & 1,7 & 2,1 & 8,6 & 7,8 & 7,1 \\
\hline
\end{tabular}

Ancrumia cuspidata

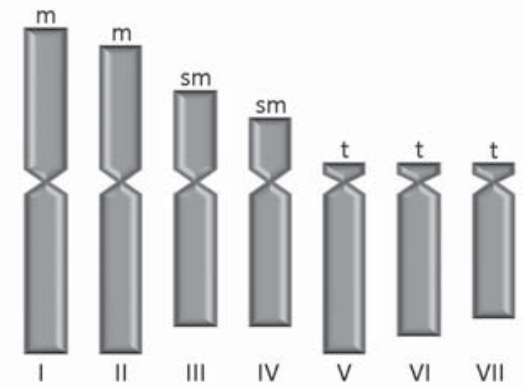

\begin{tabular}{|l|l|l|l|l|l|l|l|}
\hline BU(5) & $5,47 \pm 0,20$ & $5,20 \pm 0,31$ & $4,54 \pm 0,22$ & $4,42 \pm 0,28$ & $5,28 \pm 0,19$ & $4,85 \pm 0,25$ & $4,25 \pm 0,28$ \\
\hline
\end{tabular}

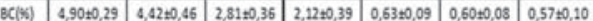
\begin{tabular}{|l|c|c|c|c|c|c|c|}
\hline $\operatorname{LR}(*)$ & 10,37 & 9,62 & 7,34 & 6,54 & 5,91 & 5,45 & 4,82 \\
\hline
\end{tabular} \begin{tabular}{|l|c|c|c|c|c|c|c|}
\hline LT( $(\mathrm{mm})$ & 14,00 & 13,00 & 10,50 & 9,50 & 8,50 & 7,00 & 6,00 \\
\hline$R$ & 1,1 & 1,2 & 1,6 & 2,1 & 8,4 & 8,0 & 7,5 \\
\hline
\end{tabular}

Gilliesia montana

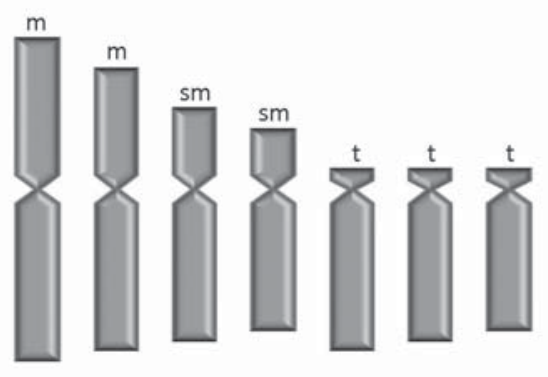

\begin{tabular}{|c|c|c|c|c|c|c|c|}
\hline & I & II & III & IV & V & VI & VII \\
\hline$u(x)$ & $5,59 \pm 0,25$ & $4,97 \pm 0,39$ & $4,84 \pm 0,35$ & $4,55 \div 0,35$ & $5,26 \pm 0,23$ & $4,86 \pm 0,28$ & $4,63 * 0,25$ \\
\hline (c) & $4,88 \pm 0,51$ & $4,26 \pm 0,31$ & $2,43 \pm 0,48$ & $1,90 \pm 0,26$ & $0,59: 0,15$ & $0,67 \pm 0,07$ & $0,58 \pm 0,12$ \\
\hline$R(x)$ & 10,47 & 9,24 & 7,26 & 6,45 & 5,85 & 5,53 & 5,20 \\
\hline$T(\mu m)$ & 12,00 & 10,50 & 8,50 & 7,50 & 7,00 & 6,50 & 5,50 \\
\hline & 1,1 & 1,2 & 2,0 & 2,4 & 8,9 & 7,3 & 8,0 \\
\hline
\end{tabular}

Speea humilis

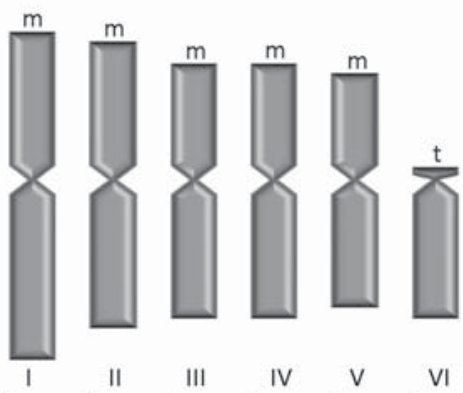

\begin{tabular}{|l|l|l|l|l|l|l|}
\hline Bu(X) & $5,98 \pm 0,25$ & $5,08 \pm 0,47$ & $4,69 \pm 0,21$ & $4,59 \pm 0,21$ & $4,27 \pm 0,11$ & $4,58 \pm 0,44$ \\
\hline
\end{tabular} \begin{tabular}{|l|l|l|l|l|l|l|l|}
\hline$B C(5)$ & $5,09 \pm 0,23$ & $4,47 \pm 0,10$ & $4,02 \pm 0,15$ & $3,77 \pm 0,18$ & $3,44 \pm 0,33$ & $0,44 \pm 0,15$ \\
\hline
\end{tabular}

\begin{tabular}{|c|c|c|c|c|c|c|}
\hline $\operatorname{LR}(\$)$ & 11,07 & 9,55 & 8,70 & 8,37 & 7,71 & 5,03 \\
\hline
\end{tabular} \begin{tabular}{|l|l|l|l|l|l|l|}
\hline IT $(\mu \mathrm{m})$ & 18,00 & 16,00 & 15,00 & 14,50 & 13,00 & 10,00 \\
\hline
\end{tabular}

Figura 3. Parte 1. 
Miersia tenuiseta

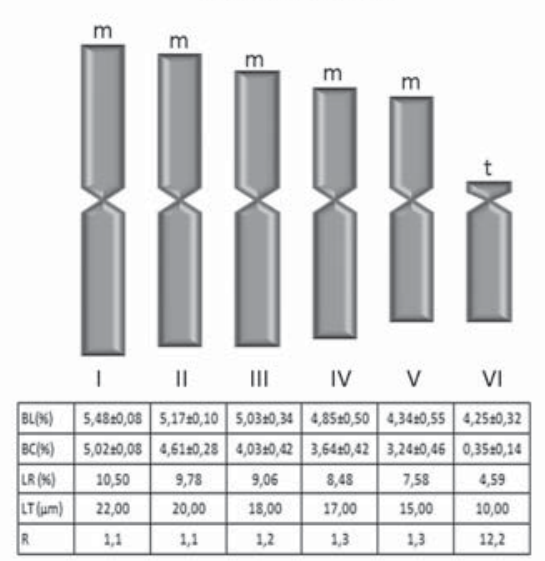

Miersia leporina

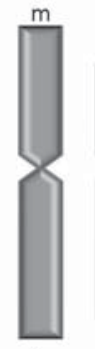

I II III IV V V

\begin{tabular}{|l|l|l|l|l|l|l|}
\hline BL(F) & $5,83 \pm 0,21$ & $5,21 * 0,31$ & $4,69 \pm 0,25$ & $4,23 \pm 0,23$ & $4,09 \pm 0,29$ & $5,19 \pm 0,24$ \\
\hline
\end{tabular}

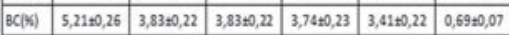

\begin{tabular}{|l|c|c|c|c|c|c|}
\hline LR(90) & 11,04 & 9,04 & 8,53 & 7,97 & 7,50 & 5,88 \\
\hline LT( $(\mu \mathrm{m})$ & 20,00 & 18,00 & 17,50 & 16,50 & 15,00 & 12,00 \\
\hline$R$ & 1,1 & 1,4 & 1,2 & 1,1 & 1,2 & 7,5 \\
\hline
\end{tabular}

Miersia minor

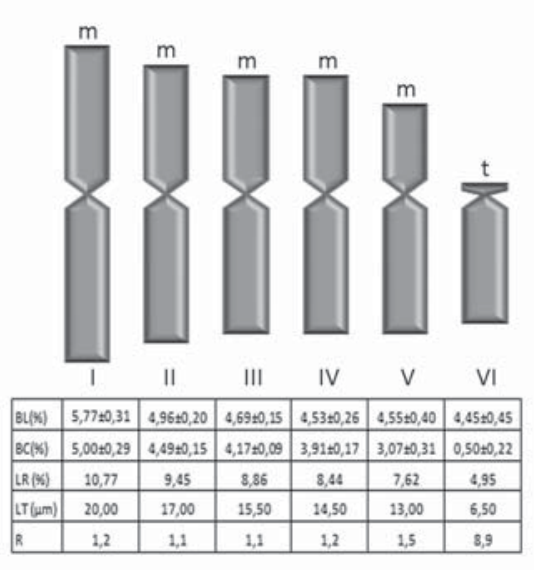

Miersia chilensis

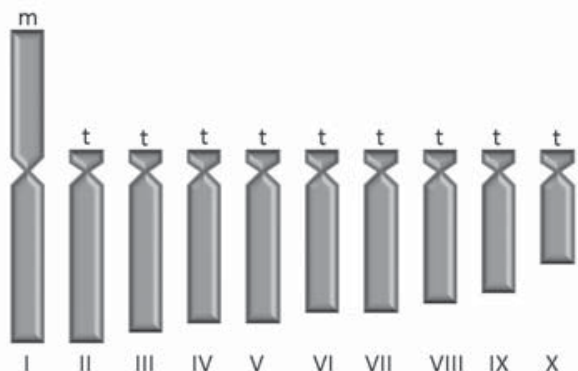

I II III IV V VI VII VIII IX $\mathrm{X}$

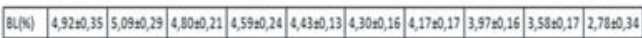

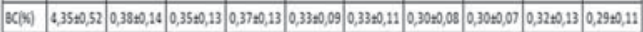
\begin{tabular}{|l|c|c|c|c|c|c|c|c|c|c|}
\hline LR(M) & 9,26 & 5,07 & 5,15 & 4,96 & 4,76 & 4,63 & 4,47 & 4,27 & 3,90 & 3,07 \\
\hline $\operatorname{LT}(\mathrm{mm})$ & 20,00 & 12,00 & 11,00 & 10,50 & 10,00 & 9,50 & 9,00 & 8,50 & 7,50 & 6,00 \\
\hline
\end{tabular}

Figura 3. Parte 2.

Figura 3. Idiogramas de los complementos cromosómicos haploides de las especies de Gilliesieae, mostrando las longitudes promedio calculadas como porcentajes (BL, brazo largo; BC, brazo corto y LR, largo relativo), longitud total del cromosoma (LT, $\mu \mathrm{m}$ ) y razón cromosómica $(\mathrm{R}=$ brazo largo/brazo corto).

FIGURE 3. Idiogram of the haploid chromosomes complement of Gilliesieae species, showing measurements calculated as percent (BL, long arm; BC, short arm and LR, relative length), total chromosome length (LT, $\mu \mathrm{m})$ and chromosome arm ratios $(\mathrm{R}=$ long /short arm).

\section{DISCUSIÓN}

Los resultados del presente estudio representan los primeros reportes sobre el cariotipo para la mayoría de las especies de la tribu Gilliesieae que crecen en Chile, constituyendo la información cariotípica más completa para el grupo (Fig. 2). Nuestros resultados son plenamente congruentes con los de Goldblatt (1976) para Miersia chilensis, reconociéndose un número cromosómico $2 \mathrm{n}=20$, con sólo 2 metacéntricos y los restantes telocéntricos, por lo cual el $2 n=12$ señalado por Del Poso \& Zöllner (1976) para esta misma especie podría corresponder a un error en la determinación del material estudiado, pudiendo tratarse de $M$. tenuiseta, dado que ambas especies coexisten y han sido consideradas sinónimos (Muñoz 2000, Escobar et al. 2010, Escobar 2012), y sobre la base de nuestros resultados presenta la misma dotación cromosómica. Sin embargo, nuestros resultados difieren parcialmente de los señalados para Solaria miersioides (Solaria attenuata, Ravenna 1967) y Gethyum atropurpureum. Ravenna (1967) señala, para Solaria miersioides, un complemento cromosómico con 4 submetacéntricos, 6 subtelocéntricos y 4 telocéntricos, contrariamente a lo observado en este estudio 
donde se muestran 4 metacéntricos, 4 submetacéntricos y 6 telocéntricos. Por otro lado, Goldblatt (1976) indica 8 cromosomasmetacéntricosen G.atropurpureum, sinembargo, en el presente estudio corresponden a 4 metacéntricos y 4 submetacéntricos. Tales diferencias estarían dadas por la interpretación del tipo de cromosomas.

La variación del número cromosómico, debido a poliploidía y disploidía, tiene clara repercusión en la filogenia y evolución del cariotipo (Guerra 2000, 2008). Mecanismos de ambas vías han sido señalados en Alliaceae, siendo común la presencia de cariotipos bimodales originados por translocaciones Robertsonianas y variados grados de ploidía (Crosa 1988, Tamura 1995, Jones 1998, Fritsch \& Friesen 2002, Pires et al. 2006, Souza et al. 2009, Souza et al. 2010, Leitch et al. 2010). Sin embargo en Gilliesieae no se observó poliploidía interpoblacional o interespecífica.

Desde un punto de vista filogenético, la estrecha relación entre Gilliesia graminea y Gethyum atropurpureum (Fay \& Chase 1996, Meerow et al. 1999, Fay et al. 2006, Escobar 2012), es apoyada por nuestros resultados, dado que ambas especies presentan la misma dotación cromosómica, por lo cual se trataría de especies evolutivamente muy relacionadas.

El tipo de cromosomas y el conservado número fundamental $(\mathrm{NF}=11)$, observado en las especies de Gilliesieae estudiadas, sugiere una evolución del cariotipo por este tipo de procesos, coincidiendo con la propuesta de Goldblatt (1976), sin embargo, contrariamente a lo propuesto por este autor, y en consideración de recientes estudios filogenéticos (Escobar 2012), nuestros resultados sugieren que la tendencia sería a aumentar el número cromosómico, por procesos de sucesivas fisiones céntricas, desde un $2 \mathrm{n}=12$ observado en Speea y Miersia a un 2n=14 en Gilliesia, Ancrumia, Gethyum y Solaria y 2n=20 en Miersia chilensis. En este contexto, los números cromosómicos señalados caracterizan los dos principales clados observados dentro de Gilliesieae (Escobar 2012). Un clado conformado por especies de Ancrumia, Gethyum, Gilliesia y Solaria con un número cromosómico $2 \mathrm{n}=14 \mathrm{y}$ presencia de tres tipos de cromosomas, caracterizado desde el punto de vista floral por una marcada simetría bilateral dada por la reducción del número de estambres (2-3) y tépalos (en Gilliesia), y un segundo clado formado por especies de Miersia y Speea, con características florales ampliamente presentes en la familia (flores con 6 tépalos, 6 estambres y simetría levemente bilateral) y un número cromosómico $2 \mathrm{n}=12$, con cromosomas mayoritariamente metacéntricos, como en representantes de la sudafricana subfamilia Tulbaghioideae (Vosa 2000), excepto por Miersia chilensis. Tal situación observada en M. chilensis, con características florales típicas de la familia y un mayor número cromosómico $(2 \mathrm{n}=20)$, con un cariotipo altamente asimétrico dominado por cromosomas telocéntricos, parece no ser exclusiva de la tribu, ya que ocurre también en representantes de la tribu hermana. Dentro de Ipheieae, Ipheion y Zoellnerallium, afín morfológicamente a Nothoscordum (6 tépalos y 6 estambres), poseen un mayor número cromosómico, $2 \mathrm{n}=20$ y 14 , respectivamente, con cromosomas predominantemente del tipo telocéntrico y mayores índices de asimetría (Crosa 2004, Souza et al. 2009, Souza et al. 2010).

Si bien la ocurrencia de translocaciones Robertsonianas parecen ser las responsables de la variación cariotípica dentro de Gilliesieae, futuros estudios que incluyan técnicas de bandeo cromosómico e hibridación podrían corroborar esta hipótesis, considerando aún más, que análisis de hibridación de sitios de bandas heterocromáticas y DNAr han contribuido a la circunscripción taxonómica y al entendimiento de variaciones cromosómicas en representantes de Ipheieae (Souza et al. 2009, Souza et al. 2010). La ocurrencia de translocaciones Robertsonianas parece común en los representantes sudamericanos de Alliaceae, a diferencia de lo señalado para las otras dos subfamilias (Fritsch \& Friesen 2002, Vosa 2000).

\section{AGRADECIMIENTOS}

Esta investigación fue posible gracias a varias personas, quienes otorgaron apoyo logístico en terreno e intercambios de información, entre ellos los investigadores Patricio Peñailillo, Nicolás García, Luiz Gustavo Rodrigues Souza y Orfeo Crosa. A los proyectos Flora de Chile, DIUC 208.111.049-1.0 y Beca CONICYT para estudios de postgrado y de apoyo a la realización de tesis doctoral.

\section{BIBLIOGRAFÍA}

Arano, H. 1963. Cytological studies in subfamily Carduoideae (Compositae) of Japan. IX. The karyotype analysis and phylogenetic considerations on Pertya and Ainsliaea. Botanical Magazine Tokyo 76: 32-39.

Arano, H. \& H. Saito. 1980. Cytological studies in family Umbelliferae 5. Karyotypes of seven species in subtribe Seselinae. La Kromosomo 2: 471-480.

Araneda, L., Salas, P. \& L. Mansur. 2004. Chromosome numbers in the Chilean Endemic Genus Leucocoryne. Journal of the American Society for Horticultural Science 129(1): 77-80.

Arroyo, M.T.K., P. Marquet, C. Marticorena, J. Simonetil, L. Cavieres, F.A. Squeo \& R. Rozzi. 2004. Chilean winter rainfall-valdivian forests. In: R.A. Mittermeier, P. Robles Gil, M. Hoffmann, J. Pilgrim, T. Brooks, C. Goettsch, J. Lamoreux \& G.A.B. da Fonseca (eds). Hotspots Revisited. pp. 99-103. CEMEX, México.

Baeza, C., J. Grau, M. Vosyka, T. Stuessy \& H. Weiss. 2000. Recuentos cromosómicos en especies de Hypochaeris L. de Chile. Gayana Botánica 57(1):105-106.

Cave, M.S. \& M.V. Bradley. 1943. Alteration of chromosome 
number in Miersia chilensis. American Journal of Botany 30(2): 142-149.

Cox, A.V., G.J. Abdelnour, M.D. Bennett \& I.J. Leitch. 1998. Genome size and karyotype evolution in the Slipper orchids (Cypripedioideae: Orchidaceae). American Journal of Botany 85(5): 681-687.

Crosa, O. 1972. Estudios cariológicos en el género Nothoscordum (Liliaceae). Boletín de Investigación, Facultad de Agronomía, Universidad de la República. Montevideo.122: 3-8.

Crosa, O. 1975. Las especies unifloras del género Nothoscordum Kunth y el género Ipheion Rafinesque de la tribu Allieae (Liliaceae). Darwiniana 19: 335-344.

Crosa, O. 1981. Los cromosomas de cinco especies del género Tristagma (Liliaceae). Darwiniana 23: 361-366.

Crosa, O. 1988. Los cromosomas de nueve especies del género chileno Leucocoryne Lindley (Allieae-Alliaceae). Boletín de Investigación, Facultad de Agronomía, Universidad de la República. Montevideo. 17:1-12.

Crosa, O. 2004. Segunda especie y justificación del género Zoellnerallium (Alliaceae). Darwiniana 42:165-168.

Del Poso, C. \& O. Zöllner. 1976. Miersia chilensis Lindley. Plant Life 32: 118-120.

Duncan, R.E. \& R.A. MacLeod. 1950. The chromosomes of Eremantha tesselata. American Orchid Society Bulletin 19: 137-142.

Escobar, I. 2012. Sistemática de la tribu Gilliesieae Lindl. (Alliaceae), sobre la base de evidencias morfoanatómicas, citológicas y moleculares. Tesis Doctoral. Universidad de Concepción. Chile.

Escobar, I., P. Novoa, E. Ruíz, M. Negritto \& C. Baeza. 2010. Nuevo hallazgo de Miersia cornuta Phil. (GilliesieaeAlliaceae). Gayana Botánica 67:130-134.

FAY, M.F. \& M. ChASE. 1996. Resurrection of Themidaceae for the Brodiaea alliance, and recircumscription of Alliaceae, Amaryllidaceae and Agapanthoideae. Taxon 45: 441451 .

FAY, M.F. \& T. HALL. 2007. Gethyum atropurpureum (Alliaceae). Curtis's Botanical Magazine 24(2): 121-126.

Fay, M.F., P.J. Rudall \& M.W. Chase. 2006. Molecular studies of subfamily Gilliesioideae (Alliaceae). Aliso 22: 367371.

Fritsch, R.M. \& N. Friesen. 2002. Evolution, Domestication and Taxonomy. In: H.D. Rabinowitch \& L. Currah (Eds.). Allium Crop Science: Recent Advances. pp. 5-30. CABI Publishing.

Goldblatt, P. 1976. New or Noteworthy Chromosome Records in the Angiosperms. Annals of the Missouri Botanical Garden 63(4): 889-895.

Guerra, M. 2000. Chromosome number variation and evolution in monocots. In: Monocots: Systematics and Evolution. CSIRO, Melbourne, Australia.

Guerra, M. 2008. Chromosome numbers in plant cytotaxonomy: concepts and implications. Cytogenetic and Genome Research 120(3-4): 339-350.

Holmgren, P.K., N.H. Holmgren \& L.C. Barnett. 1990. Index Herbariorum. Part I: The Herbaria of The World. Edition 8. New York Botanical Garden. New York. USA.

JONES, K. 1998. Robertsonian fusion and centric fission in karyotype evolution of higher plants. Botanical Review
64: 273-289.

Leitch, I.J., J.M. Beaulieu, M.W. Chase, A.R. Leitch \& M.F. Fay. 2010. Genome size dynamics and evolution in Monocots. Journal of Botany, Article ID 862516, 18 pp.

Levan, A., K. Fredga \& A. Sandberg. 1964. Nomenclature for centromeric position on chromosomes. Hereditas 52: 201-220.

Meerow, A.W., M.F. Fay, Ch.L. Guy, Li Qin-Bao, F.Q. Zaman \& M.W. ChASE. 1999. Systematics of Amaryllidaceae based on cladistic analysis of plastid $r b c \mathrm{~L}$ and $t r n \mathrm{~L}-\mathrm{F}$ sequence data. American Journal of Botany 86(9): 1325-1345.

Meric, C. \& F. Dane. 2005. Determination of ploidy levels in Ipheion uniflorum (R. C. Graham) Rafin (Liliaceae). Acta Biologica Hungarica 56(1-2):129-136.

MuÑoz, M. 2000. Consideraciones sobre los géneros endémicos de monocotiledóneas de Chile. Noticiario Mensual del Museo Nacional de Historia Natural de Chile. 343: 1627.

Myers, N., R.A. Mittermeier, C.G. Mittermeier, G.A.B. DA FonsecA \& J. Kent. 2000. Biodiversity hotspots for conservation priorities. Nature 403: 853- 858.

Negritto, M.A., E. Ruiz, S. Beck, I. Escobar \& C. Baeza. 2010. Schickendantziella trichosepala (Alliaceae), nueva cita para Bolivia. Gayana Botánica 67(1): 135-137.

Pires, J.C, I.J. Maureira, T.J. Givnish, K.J. Sytsma, O. Seberg, G. Petersen, J.I. Davis, D.W. Stevenson, P.J. Rudall, M.F. Fay \& M.W. Chase. 2006. Phylogeny, genome size, and chromosome evolution of Asparagales. Aliso 22: 287-304.

RAHn, K. 1998. Alliaceae. In: K. Kubitzki (ed.). The families and genera of flowering plants. 3: 70-78. Springer, Verlag, Berlin, Germany.

Ravenna, P. 1967. Estudios sobre Liliáceas. El género Solaria, su presencia en la flora Argentina. Boletín de la Sociedad Argentina de Botánica 11(2-3): 157-164.

Ravenna, P. 2000a. The family Gilliesiaceae. Onira 4(3): 11-14.

Ravenna, P. 2000b. Two new Trichlora species (Gilliesiaceae) from North Peru. Onira 4(9): 31-32

Ravenna, P. 2000c. New or noteworthy Miersia species (Gilliesiaceae). Onira 5(7): 31-34.

Ravenna, P. 2005a. Gilliesia dimera and G. isopetala new species from Central Chile (Gilliesiaceae). Onira 9 (16): 60-63.

Ravenna, P. 2005b. Solaria brevicoalita and S. curacavina, two new species of Chilean (Gilliesiaceae). Onira 9(17): 6467.

Reeves, A. 2001. MicroMeasure: a new computer program for the collection and analysis of citogenetic data. Genome 44: 239-443.

Rudall, P., R. Bateman, M.F. Fay \& A. Eastman. 2002. Anatomy and systematics of Alliaceae with particular reference to Gilliesia, a presumed insect mimic with strongly zygomorphic flowers. American Journal of Botany 89(12): 1867-1883.

Salas, P. \& L. Mansur. 2004. Gene flow between parents with different ploidy levels in a natural population of Leucocoryne Lindley. Journal of the American Society for Horticultural Science 129(6): 833-835.

Souza, L.G.R., O. Crosa, H. Winge \& M. Guerra. 2009. The karyotype of Nothoscordum arenarium Herter (Gilliesioideae, Alliaceae): a populational and 
cytomolecular analysis. Genetics and Molecular Biology 32: 111-116.

Souza, L.G.R., O. Crosa \& M. Guerra. 2010. Karyological circumscription of Ipheion Rafinesque (Gilliesioideae, Alliaceae). Plant Systematics and Evolution 287: 119127.
TAMURA, M.N. 1995. A karyological review of the orders Asparagales and Liliales (Monocotyledonae). Feddes Repertorium 106: 83-111.

VosA, C.G. 2000. A revised cytotaxonomy of the genus Tulbaghia (Alliaceae). Caryologia 53:83-112.

Recibido: 23.03 .12

Aceptado: 18.07.12 